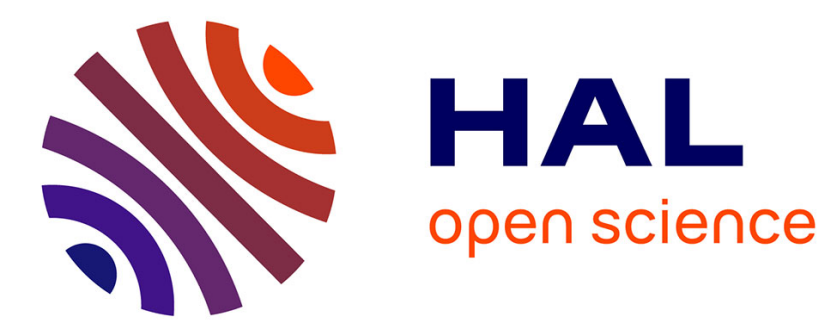

\title{
Supervision of adaptive fuzzy controllers for manufacturing systems
}

Karim Tamani, Georges Habchi, Reda Boukezzoula

\section{To cite this version:}

Karim Tamani, Georges Habchi, Reda Boukezzoula. Supervision of adaptive fuzzy controllers for manufacturing systems. CIMCA 2006, Nov 2006, Sydney, Australia. pp.CD ROM. hal-00403814

\section{HAL Id: hal-00403814 \\ https://hal.univ-smb.fr/hal-00403814}

Submitted on 8 Jan 2013

HAL is a multi-disciplinary open access archive for the deposit and dissemination of scientific research documents, whether they are published or not. The documents may come from teaching and research institutions in France or abroad, or from public or private research centers.
L'archive ouverte pluridisciplinaire HAL, est destinée au dépôt et à la diffusion de documents scientifiques de niveau recherche, publiés ou non, émanant des établissements d'enseignement et de recherche français ou étrangers, des laboratoires publics ou privés. 


\title{
Supervision of adaptive fuzzy controllers for manufacturing systems
}

\author{
*Karim Tamani, **Georges Habchi, *Reda Boukezzoula \\ *LISTIC - ESIA $\quad * *$ SYMME - ESIA \\ Domaine universitaire \\ B.P. 806 - 74016 Annecy Cedex \\ \{Karim.Tamani, Georges.Habchi, Reda.Boukezzoula\}@univ-savoie.fr
}

\begin{abstract}
The control of manufacturing systems is a problem due to its complexity and conflicting goals within the different production objective. In this article, in order to cope with some of these difficulties, we introduce a two-level fuzzy logicbased control structure, allowing the division of the complex control problem into elementary production modules. The proposed approach is characterized by two hierarchical levels. On the lower level of the hierarchy, each production module is designed as an adaptive fuzzy controller which acts independently from the others to regulate the flow of the material into a system, and on the upper level, a supervisor adjusts the parameters of the sub-controllers in order to improve the overall performance and restrict the system to the admissible domain. This leads to hierarchical and distributed control structure.
\end{abstract}

\section{Introduction}

The design and the operation of manufacturing systems are of great economic importance. Current advances in manufacturing have resulted in the improvement of manufacturing processes, but have also led to changes in manufacturing management. Concepts such as throughput, cycle time, and the in-process inventories, widely known as Work-In-Process (WIP), are traditionally some of the most important performance measures of manufacturing systems. The WIP inventory is measured by the number of unfinished parts in the buffers throughout the manufacturing system. Most manufacturing systems are large dynamic systems consisting of a network of machines and buffers that, over time, produce a variety of products that are in demand. Each product follows a given process that specifies the sequence of machines it must visit and the operations performed by them. Over time, machines are often subject to breakdowns and then to repairs.

A particular characteristic of a manufacturing system con- cerns the complexity and the presence of uncertainties along with the difficulties in building analytical models that represent the system from all its major angles. Thus, optimal control strategies, or at least good ones, are hard to find and the full potential of manufacturing systems is not completely exploited.

Substantial literature exists on control policies for manufacturing systems. This literature includes research on simulation studies; performance analysis of queuing theory, stability, and optimal controls; fluid approximations of discrete systems; and many other relevant topics. See $[8,9,11,14]$ for a survey.

However, because of the large size of these systems, the use of analytical approaches such as Petri net or supervisory theory, are not suitable for the tracking problems, and the optimal policies are extremely difficult, if not impossible, to obtain.

There are quite a few papers dealing with computational methods to obtain optimal or approximately optimal solutions. Most of them address only single machine (or parallel machine) models requiring no constraints on the state of the system; see [2] for example. Thus, it appears that obtaining optimal solutions for large, complex systems is not a realistic goal.

Since neither analytical nor computational solutions are achievable, heuristic policies, supported by fuzzy theory, are suggested to control flow within production systems. The application of fuzzy control concepts in manufacturing systems has not received much attention until recent years, and this mainly in the field of scheduling $[1,4,5,16]$. The problem that we deal in this paper is how to obtain rates of production of intermediate parts and finished products in a manufacturing system consisting of a network of resources. The objective is to meet demand for finished products, while guaranteeing stability. This is similar to the trajectory tracking problem in the control systems theory.

In order to cope with the complexity of the system, we introduce a two level fuzzy logic based control structure, allowing the division of the complex control problem into el- 


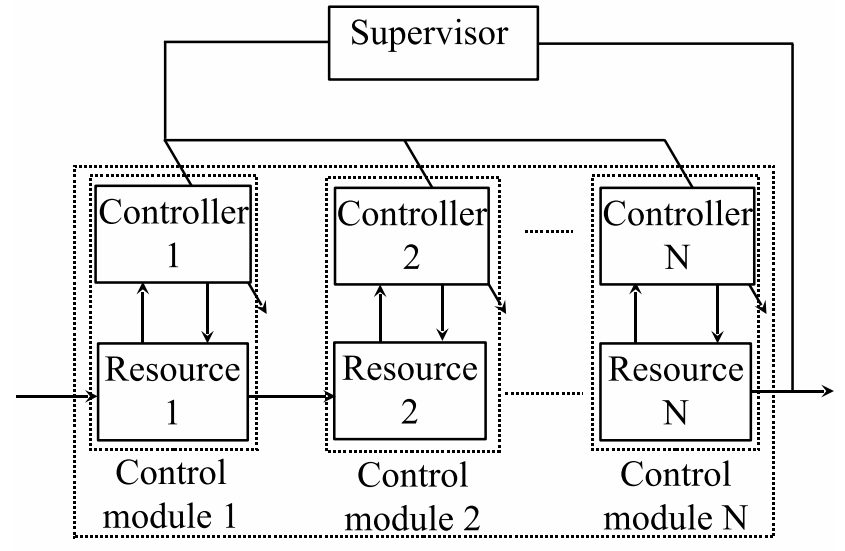

Production system

\section{Figure 1. Hierarchical control structure}

ementary production modules. This provides a distributed and hierarchical frame (Figure 1).

The proposed approach is characterized by two hierarchical levels. In the lower level, there are distributed fuzzy controllers which acts independently from each others to regulate the production flow in the system. Each decision is built according to local dynamic information. In the upper level, the supervisor has the task of coordinating and tuning the local controllers, using the performance measurements characterizing the overall system's current behavior to achieve better performance and restrict the system in admissible domain.

The remainder of this paper is organized as follows. Section 2 concerns the division of the production systems as proposed in [15]. Section 3 describes the fuzzy local controller. The supervisory control strategy is detailed in section 4 . In section 5, simulation results are given to illustrate the feasibility of the approach. Concluding remarks are given in section 6 .

\section{Division of production systems}

The proposed division is an analytical approximation technique for designing control at the elementary resources stage. According to the production floor modeling approach introduced and explained in [15], every manufacturing system may be divided into basic modules including the transfer, assembly, and disassembly module. An elementary module includes a machine $M_{i}$, and all its upstream and downstream buffers $B_{k, i}, B_{i, l}(k=1, \ldots, \mathrm{K}$ and $l=1, \ldots, \mathrm{L})$ respectively. These modules, if connected to each other, may represent manufacturing networks of various layouts.

In each module, the controller decides how "fast" the machine should produce, given the levels of its upstream and downstream buffers, and the surplus which is the difference between the current production and demand (i.e., the tracking error).

\section{Design of adaptive fuzzy controllers for a single machine}

The fuzzy control law is designed so that the following conditions are satisfied:

1. The closed loop system must be stable in the sense that all the upstream and downstream flows are bounded.

2. The tracking error converges to zero.

The first condition ensures that the demand is feasible, while the second condition keeps the production (output) close to the demand (target).

The control objective is to satisfy the demand and keep WIP as low as possible, while ensuring stability in the sense of Lyapunov by combining conditions 1 and 2 (asymptotic stability). This is attempted by constantly regulating the production rate $u_{i}$ taking the capacity constraint of each resource as well as the feasibility condition of the demand into account.

\subsection{System state variables, feasibility and stability}

In this framework, the control policy regulates the production flow into a system. The production flow is modeled as continuous, i.e., as a fluid. In process inventories (WIP), surpluses (defined below), and production rates are represented by continuous (i.e., real) variables. Here, we consider the simple case of the single part type.

The machines are unreliable and then subject to failures. We denote the state of the machine " $i$ " at time " $t$ " by a binary variable $\alpha_{i}(t)$. When the machine is down, $\alpha_{i}(t)=0$; otherwise $\alpha_{i}(t)=1$. The delay for the machine " $i$ " being down (up) follows the exponential distribution with the means of $r r_{i}^{-1}\left(p_{i}^{-1}\right)$, where $r r_{i}$ and $p_{i}$ are the repair and failure rates respectively.

The fraction of time that $M_{i}$ is operational is $e_{i}=r r_{i} /\left(r r_{i}+\right.$ $\left.p_{i}\right) . \mu_{i}=1 / \tau_{i}$ is the maximum rate at which machine $M_{i}$ can process a part, where $\tau_{i}$ is the processing time of $M_{i}$. We assume that the demand for parts is constant at rate $d>0$. Thus, the statement of feasibility in the case of the single part type is given by [6]:

$$
\mu_{i} \cdot e_{i}>d
$$

Let $u_{i}(t)$ be the instantaneous production rate of parts performed at $M_{i}$. The production rates are the control variable in this formulation. It depends on the state of the machine defined by the variable $\alpha_{i}(t)$. Then, the capacity constraints are:

if $\alpha_{i}(t)=0, u_{i}(t)=0$

if $\alpha_{i}(t)=1, u_{i}(t) \cdot \tau_{i} \leq 1 ; 0 \leq u_{i}(t) \leq \mu_{i}$ 
We define " $u_{i}(t) \cdot \tau_{i}$ " to be the fraction of the capacity of machine $M_{i}$ that is devoted to the single part type at time $t$. To ensure a stable system, and thus, the existence of feasible control policies, the feasibility statement (1) and the constraints capacity (2) must be satisfied [11, 14].

To solve the continuous-flow control problem, we discretize time and assume fixed control during each time increment. Let $t_{k}$ denote the $k^{\text {th }}$ discrete time point. The dynamic of a machine $M_{i}$ is constantly given by its production as follows:

$$
y_{i}\left(t_{k+1}\right)=y_{i}\left(t_{k}\right)+u_{i}\left(t_{k}\right)\left(t_{k+1}-t_{k}\right)
$$

Where $t_{k+1}, t_{k}$ are the times when control actions (changes in processing rates) happen. The buffer levels at any time are given by:

$$
x_{i, i+1}\left(t_{k+1}\right)=x_{i, i+1}\left(t_{k}\right)+\left[u_{i}\left(t_{k}\right)-u_{i+1}\left(t_{k}\right)\right]\left(t_{k+1}-t_{k}\right)
$$

Let us define the surplus $s_{i}\left(t_{k}\right)=y_{i}\left(t_{k}\right)-D\left(t_{k}\right)$ (where $D\left(t_{k}\right)=d \cdot t_{k}$ is the cumulative demand) as the difference between the cumulative production and demand (the tracking error). If it is negative, customers are not satisfied. If it is positive and large, there is a large finished goods inventory. Keeping the surplus near zero is a major objective.

\subsection{Fuzzy control synthesis for a single ma- chine}

In [10], the authors described a fuzzy controller for each machine with the input variables:

- The levels of the upstream and downstream buffers;

- The production surplus;

- The state of the machine.

The output variable of each controller is the processing rate of each machine. The buffer levels, surplus, and the processing rate of each machine use linguistic variables with certain membership functions.

Since the major control objective is to keep the error between the production and demand close to zero, we use, in this case, an adaptive fuzzy controller based on the Takagi-Sugeno fuzzy model [3]. The chosen approach consists in adjusting the conclusion parameter, which provides the fraction of the capacity of the machine devoted to processing.

In the case of a transfer module composed of a machine $M_{i}$, one upstream buffer, and one downstream buffer, the Takagi-Sugeno fuzzy rules describing the controller are:

$$
\begin{aligned}
& R^{\left(i_{1}, i_{2}, i_{3}\right)}: \operatorname{IF} x_{i-1, i} \text { is } X_{1}^{i_{1}} \text { AND } x_{i, i+1} \text { is } X_{2}^{i_{2}} \text { AND } s_{i} \text { is } X_{3}^{i_{3}} \\
& \text { THEN } r_{i}=\phi\left(i_{1}, i_{2}, i_{3}\right) \\
& \text { where } X_{p}^{i_{p}}(p=1,2,3) \text { is the } i_{p}^{t h} \text { linguistic term associated } \\
& \text { with the vector of the input variables } x=\left[x_{i-1, i} x_{i, i+1} s_{i}\right]
\end{aligned}
$$

which are the upstream/downstream buffer levels, and the surplus, respectively, while $\phi\left(i_{1}, i_{2}, i_{3}\right)$ denote the real value involved in the rule conclusion. Table 1 shows the fuzzy sets defined for all the input variables. We consider strict partitioning of the different inputs into the universe of discourse, with symmetric triangle-shaped membership function for each fuzzy set and uniformly distributed [12]. The buffer levels are normalized on the range [0,1], while the surplus is normalized on $[-1,1]$. The gains are used to map the actual inputs of the fuzzy system to the normalized universe of discourses.

\section{Table 1. Linguistic term of the fuzzy sets (E=Empty, A=Almost, $\mathrm{N}=$ Normal, $\mathrm{F}=\mathrm{Full}$, NEG=Negative, Z=Zero, POS=Positive)}

\begin{tabular}{|c|ccccc|}
\hline Variables & \multicolumn{5}{|c|}{ Fuzzy sets } \\
\hline$x_{i-1, i}$ & $\mathrm{E}$ & $\mathrm{AE}$ & $\mathrm{N}$ & $\mathrm{AF}$ & $\mathrm{F}$ \\
\hline$x_{i, i+1}$ & $\mathrm{E}$ & $\mathrm{AE}$ & $\mathrm{N}$ & $\mathrm{AF}$ & $\mathrm{F}$ \\
\hline$s_{i}$ & NEG & $\mathrm{Z}$ & POS & & \\
\hline
\end{tabular}

The output generated by the fuzzy controller $0 \leq r_{i} \leq$ 1 constantly "decides" how "fast" the machine $M_{i}$ should produce. It is given by:

$$
r_{i}= \begin{cases}0 & \text { if } \alpha_{i}=0 \\ \sum_{\left(i_{1}, i_{2}, i_{3}\right) \in I} \xi^{\left(i_{1}, i_{2}, i_{3}\right)}(x) \cdot \phi\left(i_{1}, i_{2}, i_{3}\right) & \text { if } \alpha_{i}=0\end{cases}
$$

where $\xi^{\left(i_{1}, i_{2}, i_{3}\right)}(x)=\prod_{p=1}^{3} \mu_{X_{p}^{i_{p}}}\left(x_{p}\right)$ represents the truth value of the premises of rules $R^{\left(i_{1}, i_{2}, i_{3}\right)}$, and $I=I_{1} \times I_{2} \times I_{3}$ the sets of labels that represent the rule base. $\mu_{X_{p}^{i_{p}}}\left(x_{p}\right)$ is the grade of the membership function of $x_{p}$ in $X_{p}^{i_{p}}$.

In compact form, the output function is simply given when $\alpha_{i}=1$ by:

$$
r_{i}=W \cdot \Phi
$$

The adaptation consists in adjusting $\Phi$ at each step so that the tracking error (i.e., surplus $s$ ) converges to zero. This is applied by using the following algorithms:

$$
\Phi\left(t_{k+1}\right)=\Phi\left(t_{k}\right)-\eta \cdot W \cdot s_{i}\left(t_{k}\right)
$$

where $\eta$ is a positive constant value.

When the tracking error is satisfied (i.e., surplus close to zero), the controllers keep buffers regulating the machines rates at neither full nor empty. In this case, the structure of the controllers is the same as described in [10].

Considering the simple case of one product with one operation, the production rate of machine $M_{i}$ would be:

$$
u_{i}\left(t_{k}\right)=\frac{r_{i}}{\tau_{i}}=r_{i} \cdot \mu_{i}
$$

As stated in [1], the choice of the saturation value $B$ (buffer sizes) for every buffer has an influence on the control performance. In the field of the fuzzy control, it defines the 


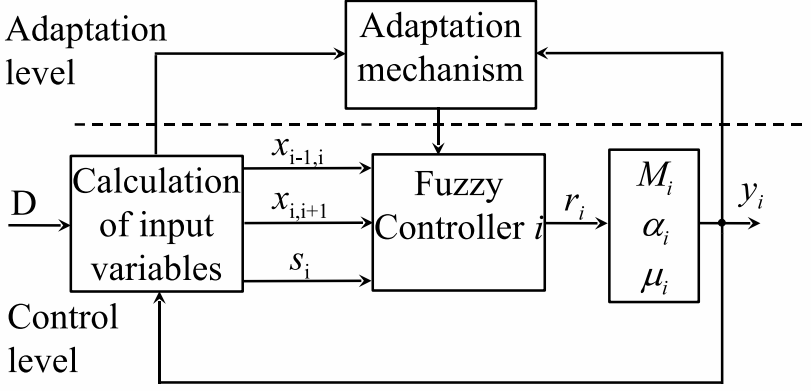

Figure 2. Adaptive fuzzy controller for single machine

universes of discourse $[0, B]$ of the buffer levels. The optimal buffer sizes are assessed by building safety stocks to react to disruptive events that occur as part of the production process, particularly repairs and failures. To resolve this problem, we use an iterative approach. The parameter $B$ is initially set to 1 . A first simulation is run with this value and the maximum levels on each buffer are used as new values to normalize the $B$ parameters for successive simulations. This procedure is repeated until the $B$ parameters converge. This processing approach leads to an adaptive fuzzy controller (Figure 2).

\section{Design of supervisory controller}

In control systems literature, a supervisor is a controller that uses actual available data to characterize the overall system's current behavior and then modify the lower level controllers to ultimately achieve the desired specification [13]. In our case, we adapt the approach proposed in [10] to tune the distributed lower-level controllers to ensure the coordination between them. This leads to the optimization of the system's operations. The supervised control structure is shown in Figure 3.

The objective of the supervisory controller is to restrict the system in the admissible domain of the final surplus; since the surplus is giving a more precise picture of the system's state. If it is negative, customers are not satisfied. If it is positive and has a high value, WIP is high.

The input variables of the supervisor are:

The mean surplus of the end product $(s)$, the error deviation $(d s)$, and the value of the mean work-in-process (wip). Both the parameters $s$ and $d s$ are used to keep production close to the demand, while the variable wip restrict the number of parts in processing. We use the relative wip error (ewip) as a measure of WIP performance in order to avoid large deviations from the mean value [10]. This is due to the fact that analytical measurements of the optimal wip cannot be assessed.
The outputs of the supervisor are the correction factors $-1 \leq u_{c} \leq 1$ and $-1 \leq l_{c} \leq 1$ of the upper and lower admissible domain (surplus) bounds, respectively. These correction factors express the percentage by which the domain's bounds are altered.

The expert knowledge that describes the supervisory control objective is built on the following assumption; adaptive surplus bounds may improve the production performance and guarantee the respect of the specification given in terms of the maximum allowable WIP. It can be summarized in the following statements:

If the WIP is high (low) and the final surplus is positive high (negative high), then reduce (increase) the upper (lower) bound of the admissible domain.

The above knowledge is formally represented as a fuzzy logic rule, as follows:

$R^{(k)}$ : IF $s$ is $S^{(k)}$ AND $d s$ is $D S^{(k)}$ AND ewip is $W^{(k)}$ THEN $u_{c}$ is $U^{(k)}$ AND $l_{c}$ is $L^{(k)}$

The crisp values of the output $u_{c}$ and $l_{c}$, of the corrections of the upper and lower surplus bounds, respectively, are given by the following defuzzification formulas:

$$
u_{c}^{*}=\frac{\sum u_{c} \cdot \mu_{U}^{*}\left(u_{c}\right)}{\sum \mu_{U}^{*}\left(u_{c}\right)}, l_{c}^{*}=\frac{\sum l_{c} \cdot \mu_{L}^{*}\left(l_{c}\right)}{\sum \mu_{L}^{*}\left(l_{c}\right)}
$$

where $\mu_{U}^{*}\left(u_{c}\right)$ and $\mu_{L}^{*}\left(l_{c}\right)$ are the membership functions of the upper and lower surplus bounds, respectively, which are the aggregated outcome of the fuzzy inference procedure. Using these correction factors, the admissible domain bounds are modified according to the following mechanism:

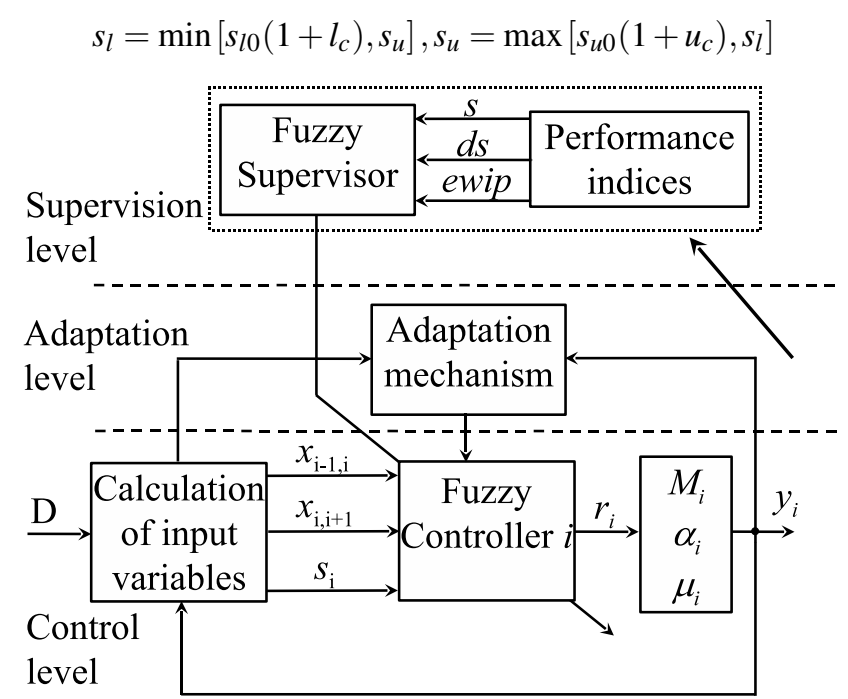

Figure 3. Supervised control structure 
where $s_{l 0}$ and $s_{u 0}$ are the lower and upper bound of the initial domain given in the specification.

Table 2 shows the fuzzy sets defined for all the variables included in the supervisory controller. The membership functions for each fuzzy set of the input variables $(s, d s$, ewip) are triangular except at the extreme right and left where they are trapezoidal. The different output $\left(l_{c}\right.$ and $\left.u_{c}\right)$ universes of discourse have symmetric triangle-shaped membership function for each fuzzy set and are uniformly distributed. All the input and output variables are normalized into the closed interval $[-1,1]$.

\section{Table 2. Linguistic terms of the fuzzy sets ( $\mathrm{N}=$ Negative, $\mathrm{B}=\mathrm{Big}, \mathrm{S}=\mathrm{Small}, \mathrm{Z}=\mathrm{Zero}$, P=Positive)}

\begin{tabular}{|c|ccccc|}
\hline Variables & \multicolumn{5}{|c|}{ Fuzzy sets } \\
\hline$s$ & NB & NS & Z & PS & PB \\
\hline$d s$ & N & Z & P & & \\
\hline ewip & N & Z & P & & \\
\hline$u_{c}$ & NB & NS & Z & PS & PB \\
\hline$l_{c}$ & NB & NS & Z & PS & PB \\
\hline
\end{tabular}

\section{Simulation testing and results}

The supervised control approach is tested in the example of the assembly line presented in [10] (Figure 4). Three cases are considered. The distributed and supervised cases with limited buffer capacities and the supervised adaptive case tune the buffer saturation levels.

The system under consideration consists of five machines producing one type of product. The failure and repair rates are equal for all machines. The repair rates are $r r_{i}=0,5$ and the failure rates are $p_{i}=0,05$. The processing times $\tau_{i}$ $(i=1, \ldots, 5)$ are chosen as follows:

$$
\tau_{1}=2, \tau_{2}=5, \tau_{3}=2, \tau_{4}=1, \tau_{5}=3
$$

All experiments have been carried out using MATLAB's FlouLib toolbox, developed in our laboratory [7], and Simulink. The duration of each simulation run is 10000 time units.

Comparative results for the mean WIP for various demand patterns are shown in Table 3 and Figure 5. In the cases of distributed and supervised control, all buffer capacities are fixed to 10. In Figure 6 the evolution of the mean WIP for the three cases in a simulation run of 10000 time units is presented.

The following observations were made, based on the results obtained.

The supervised approach achieves a substantial reduction of WIP compared with the distributed control. This is to be expected, since the main objective of the supervisor is to reduce the overall WIP. The adaptive control performs best by

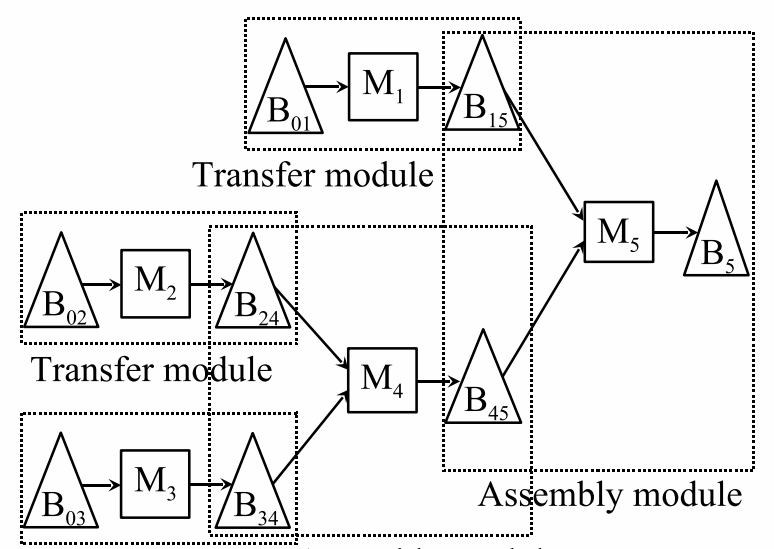

Transfer module Assembly module

Figure 4. The assembly production line

\section{Table 3. Results for the assembly line test} case

\begin{tabular}{|l|l|l|l|}
\hline $\begin{array}{l}\text { Demand } \\
\text { (parts/t.u.) }\end{array}$ & $\begin{array}{l}\text { Distributed } \\
\text { control } \\
(\mathrm{B}=10)\end{array}$ & $\begin{array}{l}\text { Supervised } \\
\text { control } \\
(\mathrm{B}=10)\end{array}$ & $\begin{array}{l}\text { Adaptive } \\
\text { control }\end{array}$ \\
\cline { 2 - 4 } & Mean WIP & Mean WIP & Mean WIP \\
\hline 0,05 & 5,543 & 2,02 & 0,886 \\
0,08 & 6,981 & 3,392 & 2,004 \\
0,1 & 7,722 & 4,155 & 2,014 \\
0,15 & 8,695 & 6,192 & 3,347 \\
0,18 & 14,08 & 14,46 & 4,934 \\
0,2 & 19,96 & 20 & 183,1 \\
\hline
\end{tabular}

optimizing the buffer capacities when the feasibility condition (1) is respected. In the three studied cases, the tracking error converges to zero, except for the case of demand $d=0,2$ parts/t.u., where the feasibility condition (1) is not satisfied for the machine $M_{2}\left(e_{2}=r r_{2} /\left(r r_{2}+p_{2}\right)=0,18<d\right)$. This leads to instability in the system. It may be seen more clearly in the case of the adaptive control by the increase of the mean WIP. This is due to the fact that the saturation value $B$ for every buffer does not converge (see Table 3 ).

\section{Conclusions}

A two-level supervised control structure based on the fuzzy theory has been presented. At the low level, the fuzzy controller is designed for each production module which is composed of one machine and its upstream and downstream buffers. The control objectives are to satisfy the demand and keep WIP as low as possible, while ensuring stability. To achieve this objective, we have used the adaptive fuzzy controller based on the Takagi-Sugeno fuzzy model. The chosen approach consists in adjusting the conclusion 


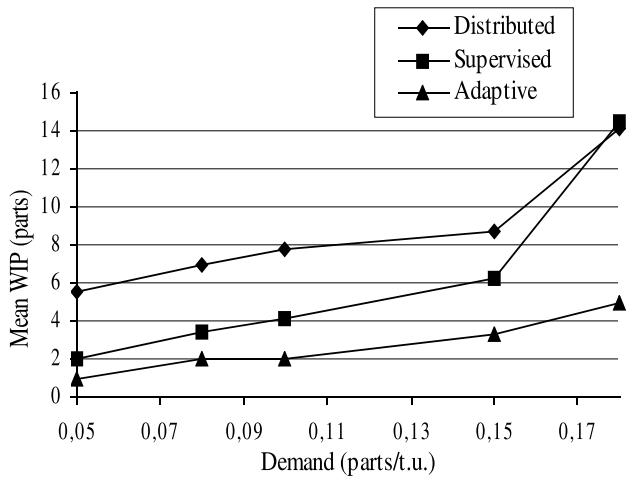

\section{Figure 5. Comparative results of mean WIP with various demands}

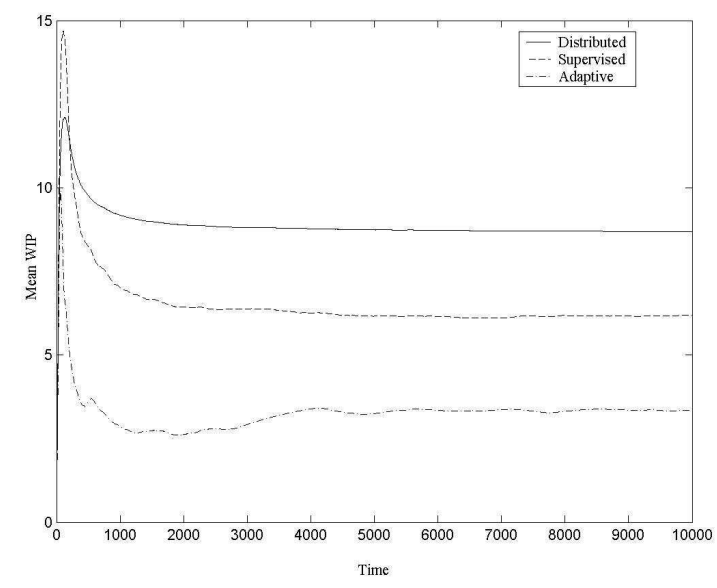

Figure 6. Evolution of mean WIP in the assembly production line with demand $d=0,15$

parameter (processing rate $r_{i}$ ). The influence of the buffer capacities have been investigated by introducing a mechanism to adjust the saturation level, based upon the maximum value of the buffer levels reached during the simulation run. The control is distributed, in the sense that each decision is made on the basis of local dynamic information alone. So, we have introduced the supervisor that uses actual available data to characterize the system's overall current behavior and then to modify the lower level controllers to ultimately achieve the desired specification. The overall control architecture has been implemented and tested for the case of a production assembly line. The results of the experiments show an improvement in performance in terms of the average WIP using adaptive control.

In future work, it would be interesting to consider the case of multi-objectives, including low production lead time, high resource utilization, few delays in delivery, etc. This leads to multi-criteria aspects of the control.

\section{References}

[1] A. Angsana and K.M. Passino. Distributed Fuzzy Control of Flexible Manufacturing Systems. IEEE Transactions on Control Systems Technology, 2(4):423-435, December 1994.

[2] X. Bai and S. B. Gershwin. Scheduling Manufacturing Systems with Work-In-Process Inventory Control: Single-PartType Systems. IIE Transactions, 27:599-617, 1995.

[3] R. Boukezzoula, S. Galichet, and L. Foulloy. Fuzzy Adaptive Control for Nonlinear Systems. Real time implementation for a robot wrist. IEEE conference on decision and control, 5:4364-4369, Florida USA, 2001.

[4] L.M.M. Custodio, J.J.S. Sentieiro, and C.F.G. Bispo. Production planning and scheduling using a fuzzy decision system. IEEE Transactions on Robotics and Automation, 10(2):160168, April 1994.

[5] P. Dadone. Fuzzy Control of Flexible Manufacturing Systems. Master of Science thesis, Blacksburg, Virginia, 1997.

[6] Y. Feng and B. Xiao. Optimal threshold control in discrete failure-prone manufacturing system. IEEE Transactions on Automatic and Control, 47(7):1167-1174, July 2002.

[7] L. Foulloy, R. Boukezzoula, and S. Galichet. An Educational Tool for Fuzzy Control. IEEE Transactions on Fuzzy Systems, 14(2):217-221, April 2006.

[8] S. B. Gershwin. Design and Operation of Manufacturing Systems: The control point policy. IIE Transactions, 32(2):891-906, October 2000

[9] G. Habchi and C. Berchet. A model for manufacturing systems simulation with control dimension. Simulation Modelling Practice and Theory, 11:21-44, 2003.

[10] S. Ioannidis, N.C. Tsourveloudis, and K. Valavanis. Fuzzy supervisory control of manufacturing systems. IEEE Transactions on Robotics and Automation, 20(3):379 - 389, June 2004.

[11] P.R. Kumar and T. I. Seidman. Dynamic Instabilities and Stabilization Methods in Distributed Real-Time Scheduling of Manufacturing Systems. IEEE Transactions on Automatic Control, 35(3):289-298, March 1990.

[12] C. C. Lee. Fuzzy Logic in Control Systems: Fuzzy Logic Controller- Part I. IEEE Transactions on Systems, Man, and Cybernetics, 20(2):404-418, March/April 1990.

[13] K.M. Passino and S. Yurkovish. Fuzzy Control. CA: Addison-Wesley Longman, Menlo Park, 1998.

[14] J. Perkins and P. R. Kumar. Stable Distributed Real-Time Scheduling of Flexible Manufacturing/Assembly/Disassembly Systems. IEEE Transactions on Automatic Control, 34(2):139-148, February 1989.

[15] N.C. Tsourveloudis, E. Dretoulakis, and S. Ioannidis. Fuzzy work-in-process inventory control of unreliable manufacturing systems. Information Sciences, 127(1-2):69-83, 2000.

[16] M.N. Yuniarto and A.W. Labib. Optimal control of an unreliable machine using fuzzy-logic control: from design to implementation. International Journal of Production Research, 43(21):4509-4537, November 2005. 